\title{
The middle communicating artery: a novel fourth-generation bypass for revascularizing trapped middle cerebral artery bifurcation aneurysms in 2 cases
}

\author{
Fabio A. Frisoli, MD, Joshua S. Catapano, MD, Jacob F. Baranoski, MD, and \\ Michael T. Lawton, MD
}

Department of Neurosurgery, Barrow Neurological Institute, St. Joseph's Hospital and Medical Center, Phoenix, Arizona

The anterior and posterior communicating arteries are natural connections between arteries that enable different adjacent circulations to redistribute blood flow instantly in response to changing supply and demand. An analogous communication does not exist in the middle cerebral circulation. A middle communicating artery (MCoA) can be created microsurgically between separate middle cerebral artery (MCA) trunks, enabling flow to redistribute in response to changing supply and demand. The MCoA would draw blood flow from an adjacent circulation such as the external carotid circulation. The MCoA requires the application of fourth-generation techniques to reconstruct bi- and trifurcations after occluding complex MCA trunk aneurysms. In this report, the authors describe two recent cases of complex MCA biand trifurcation aneurysms in which the occluded efferent trunks were revascularized by creating an MCoA.

The first MCoA was created with a "double-barrel" superficial temporal artery- $\mathrm{M}_{2}$ segment bypass and end-to-end reimplantation of the middle and inferior MCA trunks. The second MCoA was created with an external carotid artery-radial artery graft- $\mathrm{M}_{2}$ segment interpositional bypass and end-to-side reimplantation of the inferior trunk onto the superior trunk. Both aneurysms were occluded, and both patients experienced good outcomes.

This report introduces the concept of the MCoA and demonstrates two variations. Angioarchitectural and technical elements include the donation of flow from an adjacent circulation, a communicating bypass, the application of fourth-generation bypass techniques, and a minimized ischemia time. The MCoA construct is ideally suited for rebuilding bi- and trifurcated anatomy after trapping or distally occluding complex MCA aneurysms.

https://thejns.org/doi/abs/10.3171/2020.4.JNS193412

KEYWORDS bifurcation aneurysm; bypass; double-barrel; end-to-end; end-to-side; interpositional; middle communicating artery; trifurcation; vascular disorders

$\mathrm{T}$ $\mathrm{HE}$ anterior and posterior communicating arteries are natural connections between arteries that enable distinct, adjacent circulations to redistribute blood flow instantly in response to changing supply and demand. The anterior communicating artery (ACoA) joins the left and right anterior cerebral arteries (ACAs) to shift blood flow from one side to the other, whereas the posterior communicating artery (PCoA) joins the internal carotid artery (ICA) and posterior cerebral artery (PCA) to shift blood flow from front to back between the anterior and posterior circulations. These simple communications are extraordinary because they do not compromise native flow in the parent arteries; they remain quiescent but ready when needed; and they respond immediately to demands created by pressure gradients or arterial occlusions, which are often remote from and not intrinsic to the communication. The combination of one ACoA and two PCoAs to form the circle of Willis is a marvel of engineering and evolution that has prevented countless strokes and no doubt contributed to our survival as a species. It is surprising, then, that analogous communication does not exist in the middle cerebral circulation, especially when this circulation supplies critical motor, sensory, and language functions. And yet, it is challenging even to imagine how a middle communicating artery (MCoA) might look.

The answers come from deconstructing the angioarchitecture of the ACoA and PCoA. If these communications were absent, a bypass surgeon might recreate them

ABBREVIATIONS $A_{1}=$ precommunicating segment of the $A C A ; A C A=$ anterior cerebral artery; $A C o A=$ anterior communicating artery; ECA = external carotid artery; EC-IC $=$ extracranial-intracranial; ICA = internal carotid artery; ICG = indocyanine green; IC-IC = intracranial-intracranial; $M_{1}=$ sphenoidal segment of the $M C A ; M_{2}=$ insular segment of the MCA; $M C A=$ middle cerebral artery; $\mathrm{MCOA}=$ middle communicating artery; $\mathrm{mRS}=$ modified Rankin Scale; $\mathrm{PCA}=$ posterior cerebral artery; $\mathrm{PC} \mathrm{A}=$ posterior communicating artery; RAG = radial artery graft; SCA = superior cerebellar artery; STA = superficial temporal artery; TpolA = temporopolar artery.

SUBMITTED December 17, 2019. ACCEPTED April 14, 2020.

INCLUDE WHEN CITING Published online July 10, 2020; DOI: 10.3171/2020.4.JNS193412. 
with side-to-side anastomosis. For example, an in situ bypass joining the $A_{2}$ or $A_{3}$ segments brings together donor and recipient arteries that run parallel and in proximity to one another, imitating an ACoA in function, if not in form, in a position distal to the natural ACoA. However, an analogous in situ bypass for the PCoA does not exist because the ICA and PCA lack parallelism and proximity. An intracranial-intracranial (IC-IC) interpositional bypass with a graft and two anastomoses would mimic the PCoA. However, a more natural construct might take an expendable branch from both the anterior and posterior circulations and reimplant them to each other end to end. For example, a large temporopolar artery (TpolA) from the proximal $\mathrm{M}_{1}$ segment (sphenoidal segment of the middle cerebral artery [MCA]) joined to a duplicated superior cerebellar artery (SCA) with end-to-end reimplantation would look and act like a PCoA and would require just one anastomosis without an interposition graft. ${ }^{1,2}$ This TpolA-SCA bypass is an unusual application of a reimplantation technique that reattaches a recipient artery to an adjacent donor artery using an unconventional end-toend anastomosis instead of the traditional end-to-side reimplantation. Moreover, this unconventional TpolA-SCA bypass plays an unconventional role in which the reimplanted arteries may be donors rather than recipients of flow. Reimplantation with end-to-end anastomosis is an example of a fourth-generation bypass technique because the bypasses are built with unconventional structure and unconventional anastomoses. ${ }^{3,4}$

An MCoA might be constructed on the basis of similar concepts. The MCoA would be a connection between separate MCA trunks that enables flow from different parts of this territory to redistribute in response to changing supply and demand. The MCoA would draw blood flow from an adjacent circulation, such as the external carotid circulation. The MCoA would also require the application of fourth-generation techniques. An MCoA would be used to reconstruct bi- and trifurcations after occluding complex aneurysms in MCA trunks. In this report, we describe two recent cases of complex MCA bi- and trifurcation aneurysms whose occluded efferent trunks were revascularized by creating an MCoA. These case examples introduce the angioarchitecture of the MCoA and the value of this construct with combination bypasses (i.e., double and triple bypasses).

\section{Case Reports Case 1}

A 60-year-old right-handed woman with a history of smoking and an uncle and grandmother with ruptured cerebral aneurysms presented with an incidentally found left MCA trifurcation aneurysm (Fig. 1). The superior trunk arose proximal to the fusiform multilobed aneurysm, whereas the middle and inferior trunks originated from the aneurysm and had ectatic and diseased proximal segments. The patient underwent DSA, at which time the neurointerventional team deemed the aneurysm not amenable to endovascular treatment. Robust frontal and parietal branches of the superficial temporal artery (STA) were noted. Consequently, the surgical plan was a "dou-
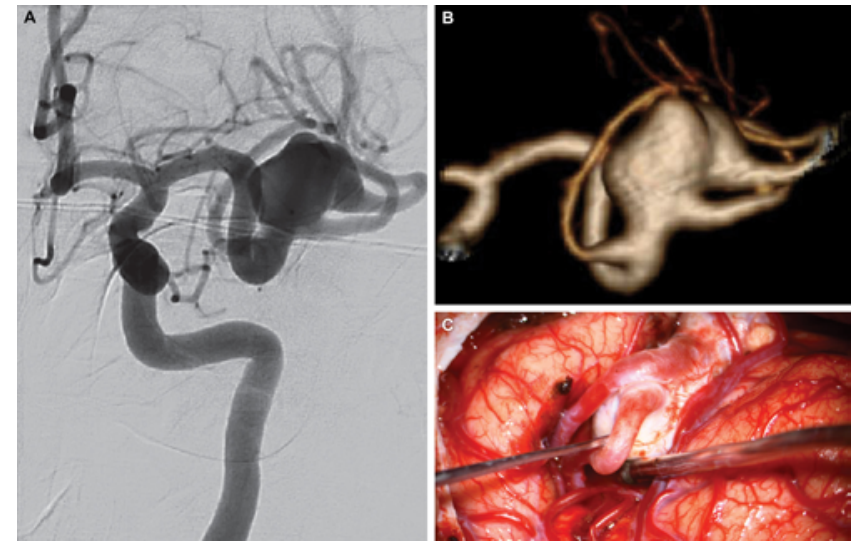

FIG. 1. Case 1. A: Left ICA injection angiogram demonstrating a large fusiform MCA trifurcation aneurysm incorporating the origin of two $\mathrm{M}_{2}$ branches. B: Digital 3D reconstruction of a CT angiogram. C: Intraoperative photograph demonstrating the aneurysm and its efferent branches. Panel $\mathrm{C}$ is used with permission from Barrow Neurological Institute, Phoenix, Arizona.

ble-barrel" end-to-side STA-M $\mathrm{M}_{2}$ (insular segment of the MCA) bypass to the middle and inferior MCA trunks and clip-trapping the aneurysm.

The STA branches were harvested microsurgically, and a pterional craniotomy was performed. The aneurysm was exposed through a transsylvian approach. Intraoperatively, both $\mathrm{M}_{2}$ branches were noted to have a large caliber, and the STA-MCA bypasses were performed successfully. There was concern that these mismatched STA donors might provide insufficient flow in isolation, and the decision was made to transect both $\mathrm{M}_{2}$ trunks from the aneurysm, proximal to the inflow of the bypass, and anastomose them to each other end-to-end (end-to-end reimplantation; Fig. 2). This anastomosis (type 4B) created an MCoA and allowed the two donor arteries to supply the new communication between inferior and middle MCA trunks, redistributing blood flow through the MCoA to either division as dictated by cerebral demand. The end-to-end anastomosis was performed with intraluminal suturing or the in situ technique on the back wall (type 4A bypass). Indocyanine green (ICG) videoangiography confirmed patency of the two STA-M $\mathrm{M}_{2}$ bypasses and the MCoA.

The patient tolerated the procedure without complication. Postoperative angiography confirmed patency of the MCoA and its donor bypasses. The patient was discharged on postoperative day 3 and made an excellent recovery (modified Rankin Scale [mRS] score of 1 at 6 months after the operation).

\section{Case 2}

A 61-year-old right-handed woman with a history of an unruptured giant left MCA bifurcation aneurysm had undergone a "picket fence" clip reconstruction in 2014. . $^{5}$ A 5-year surveillance angiogram revealed substantial aneurysm regrowth opposite the picket fence, suggesting the entire segment of the vessel was diseased (Fig. 3 ). The origins of both $\mathrm{M}_{2}$ divisions arose from the aneurysm. Consequently, the best treatment option was to trap the segment and revascularize the MCA territory with a 

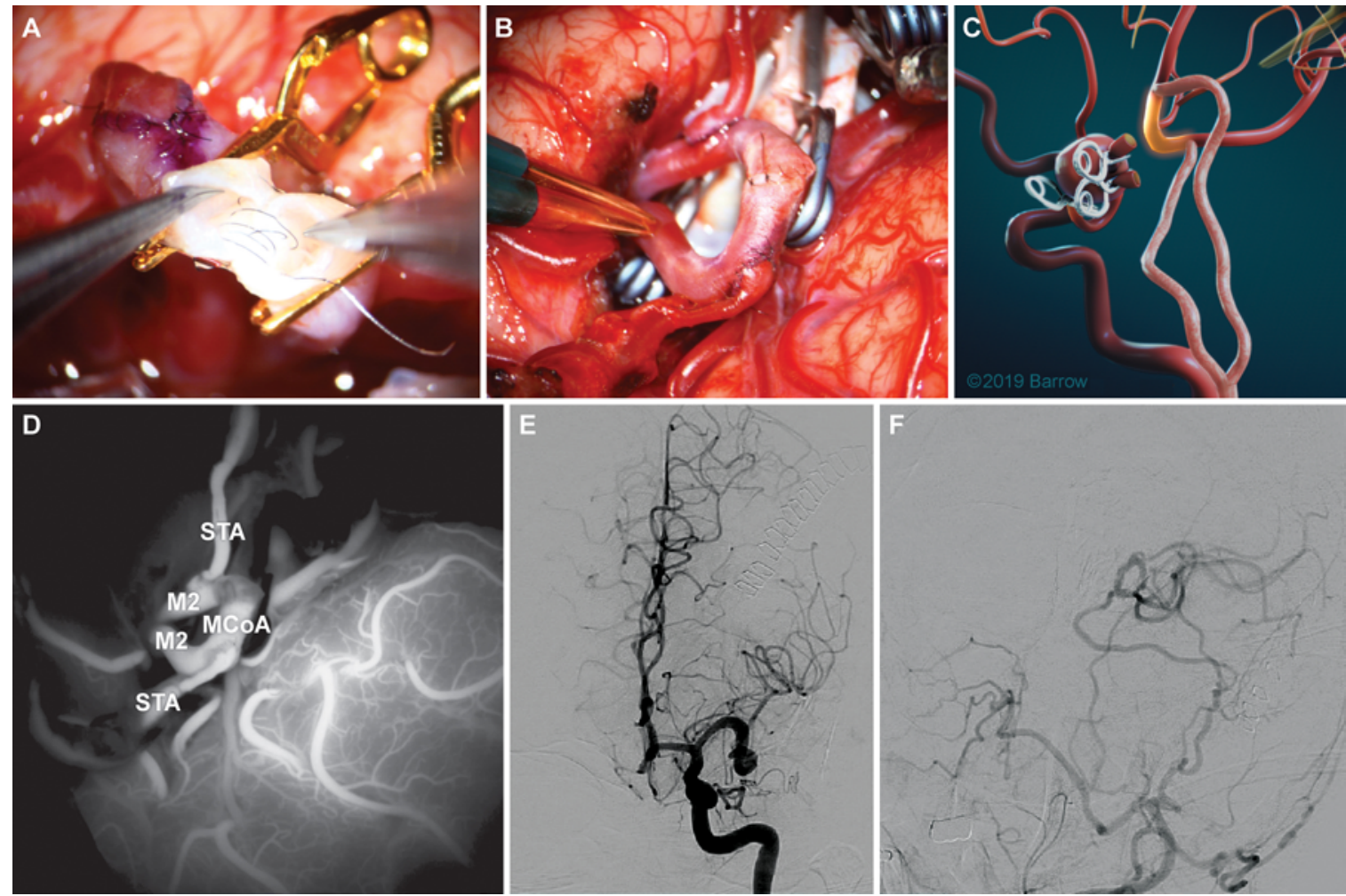

FIG. 2. Case 1. A: Intraoperative photograph demonstrating the intraluminal suturing technique used for the end-to-end anastomosis. B: Intraoperative photograph obtained after completion of the three anastomoses: two STA-M end to side and one $M_{2}-M_{2}$ end to end. C: Illustration demonstrating the final configuration of the bypass and aneurysm trapping, with the MCoA highlighted. D: Intraoperative ICG angiography demonstrating patency of the three anastomoses. E: Postoperative ICA injection DSA demonstrating obliteration of the aneurysm with preservation of the proximal $\mathrm{M}_{2}$ division. F: Postoperative ECA injection DSA demonstrating patency of the double-barrel STA-M $\mathrm{M}_{2}$ bypass with distal flow and proximal flow into the MCoA. Panels A-C are used with permission from Barrow Neurological Institute, Phoenix, Arizona.

high-flow bypass. The initial plan was to revascularize the MCA territory with a double reimplantation bypass using the external carotid artery (ECA) as a cervical donor and reimplanting the inferior and superior divisions of $\mathrm{M}_{2}$ to a radial artery graft (RAG) with end-to-side anastomoses.

A pterional craniotomy was performed, and the aneurysm was exposed through a transsylvian approach. The proximal ECA-RAG end-to-side anastomosis was performed first to arterialize the graft. Despite harvesting a $20-\mathrm{cm}$ graft, its length was insufficient to perform the double reimplantation. Therefore, the decision was made to perform a simple ECA-RAG-M 2 bypass and create the MCoA. The distal RAG was connected to the inferior $\mathrm{M}_{2}$ trunk, and the proximal $\mathrm{M}_{2}$ trunk was transected from the aneurysm. This end was then reimplanted end to side onto a branch of the superior $M_{2}$ trunk. The trunk bifurcated immediately after its origin from the aneurysm, which prevented its transection from the aneurysm and end-toend reimplantation, as was done in case 1 . The end-to-side anastomosis was performed with intraluminal suturing or the in situ technique on the back wall (type 4A bypass). The MCoA was created between the efferent trunks, the superior trunk was clip-occluded, and patency was confirmed with ICG videoangiography (Fig. 4). The aneurysm could not be proximally occluded because of the lenticulostriate arteries arising from the back of the bifurcation, which would have been trapped with a proximal $\mathrm{M}_{1}$ clip. As a result, only distal clip occlusion was performed.

The patient tolerated the procedure without complication. Postoperative angiography confirmed patency of the $\mathrm{MCoA}$ and its donor bypasses. The aneurysm no longer filled, and the lenticulostriate arteries were preserved. The patient was discharged on postoperative day 3 and made an excellent recovery (mRS score of 1 at 6 months after the operation).

\section{Discussion}

This report introduces the concept of an MCoA and demonstrates two variations. The angioarchitecture of the MCoA comprises the key elements of 1) donation from adjacent circulation, 2) a communicating bypass, 3) application of fourth-generation bypass techniques, and 4) a minimized ischemia time.

\section{Donation From Adjacent Circulation}

When the treatment of an MCA bifurcation aneurysm requires trapping or occlusion, the first consideration is replacing lost flow. The MCoA constructs that we describe received flow from the extracranial carotid circulation, in the first case via the STA branches and in the second case via the ECA (Fig. 5). These donor sources are adjacent to 

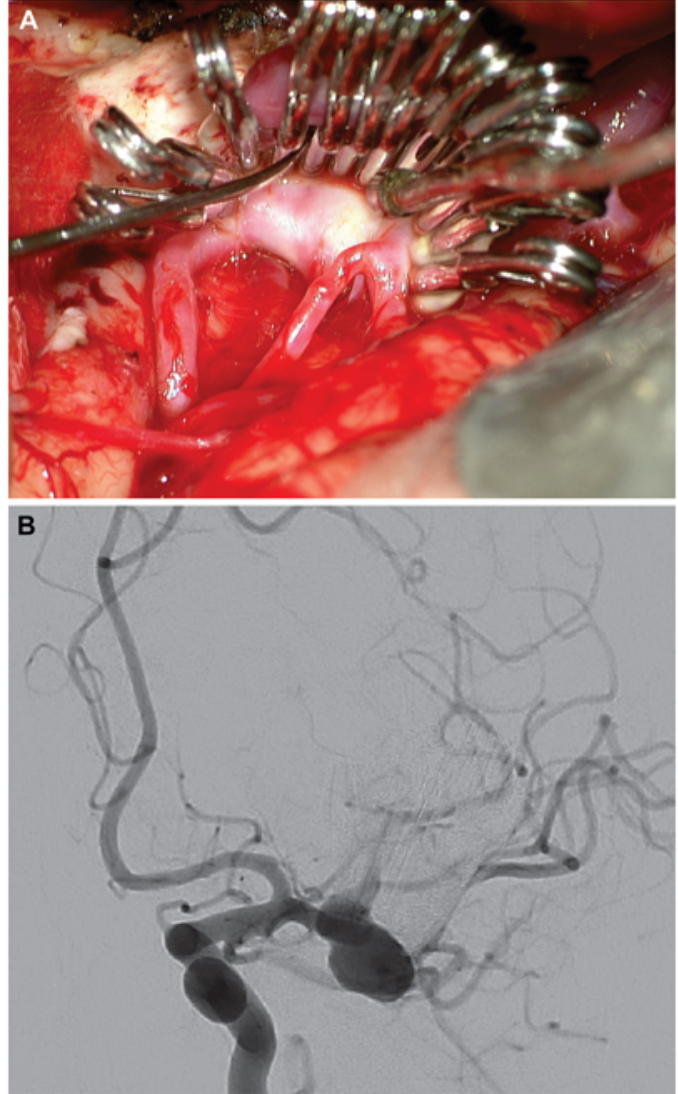

FIG. 3. Case 2. A: Intraoperative photograph of the prior picket fence clip reconstruction performed on this patient in 2014. B: ICA injection DSA demonstrating a large fusiform recurrence at the bifurcation incorporating the origins of the $\mathrm{M}_{2}$ divisions. Panel $\mathrm{A}$ is used with permission from Barrow Neurological Institute, Phoenix, Arizona.

and independent from the MCA circulation. The lateral lenticulostriate arteries make the $\mathrm{M}_{1}$ difficult to temporarily occlude for a bypass within its circulation. These basal ganglia perforators have a low tolerance to occlusion, and temporary $\mathrm{M}_{1}$ occlusion shuts off flow to the entire MCA territory. Furthermore, distal anastomoses already require temporary occlusion time for their creation, and use of the $\mathrm{M}_{1}$ as a donor would require a second round of temporary occlusion time and ischemia. The use of an adjacent donor circulation such as the ECA or its STA branches keeps MCA ischemia to a minimum. ${ }^{6,7}$ The extracranial carotid circulation can be accessed at other points, including the common carotid artery and internal maxillary artery. The proximity and accessibility of the extracranial carotid circulation make it ideal for incorporation into MCoA constructs. Other adjacent circulations include the ACA and PCA territories with donor sites at the $\mathrm{A}_{1}$ (precommunicating segment of the ACA) and $\mathrm{P}_{2}$ (postcommunicating segment of the PCA), respectively. These are deep and more technically challenging bypasses; nonetheless, we recently performed an $\mathrm{A}_{1}-\mathrm{RAG}-\mathrm{M}_{2}$ interpositional bypass with $\mathrm{M}_{2}-\mathrm{M}_{2}$ end-to-end reimplantation (not shown). Tapping adjacent circulations is a technique borrowed from embryology. Persistent trigeminal, otic, hypoglossal, and proatlantal arteries are uncommon vestigial remnants of the embryonic circulation formed by transverse anastomoses between the carotid and vertebrobasilar circulations that fail to regress during embryonic maturation.

\section{Communicating Bypass}

Once the donor flow is tapped, the MCoA must distribute it to efferent trunks. Microsurgical anastomosis joins arteries together in only three ways. Classically, the end-to-side anastomosis is a convergence bypass that redirects donor flow destined for another territory into the recipient to augment or replace lost flow, just as an STAMCA bypass redirects scalp flow into the $\mathrm{M}_{4}$ (cortical segment of the MCA). This construct is the opposite of an arterial bifurcation in which trunks divide into diverging branches that distribute blood flow peripherally. The endto-end anastomosis is a reconstructive bypass between afferent and efferent ends of an artery after the intervening pathology has been excised. Reanastomosis connects arterial ends without introducing a new donor artery and restores blood flow exactly as before, unlike a convergent bypass, which increases flow. The side-to-side anastomosis is a communicating bypass between two arteries that joins two separate arteries without compromising their inflow or outflow and without governing flow across their connection. Flow across a side-to-side anastomosis is governed by pressure gradients and can vary in its direction and degree in response to changing hemodynamics. The side-to-side anastomosis lacks a structural donor limb with a dedicated input of blood flow.

These classic concepts in microsurgical anastomosis are upended when joining trunks of a trapped bifurcation in an MCoA. There is no clear donor and recipient, and any of the three anastomoses can function as a communicating bypass. For example, the end-to-end anastomosis in case 1 and the end-to-side anastomosis between the $\mathrm{M}_{2}$ trunks in case 2 both became communicating rather than reconstructive and convergent bypasses, respectively. Therefore, the MCoA has, at its core, communication between MCA trunks with any of the three anastomoses. As with other communicating bypasses, the MCoA flow is governed by the pressure gradient: in case 1, it was the relative strength of the two STA-MCA bypasses and the demands of the recipient territories. In case 2, it was the selection of the temporal trunk as the implantation site for the interpositional RAG. In both cases, the MCoA redistributed the flow to the frontal and temporal trunks in proportion to pressure gradient and metabolic demands. The MCoA also provided some protection against bypass occlusion in case 1: if either one of the STA-MCA bypasses occluded, the remaining bypass would have supplied the other trunk via the MCoA.

\section{Fourth-Generation Bypass Techniques}

MCoA angioarchitecture requires joining MCA trunks by any means possible, with conventional or unconventional constructs and conventional or unconventional techniques, which makes it a prototypical fourth-generation bypass. The fourth-generation bypass concept was recently introduced in its historical context: first-generation bypasses are extracranial-intracranial (EC-IC) bypasses with end-to-side anastomosis between scalp arteries and 

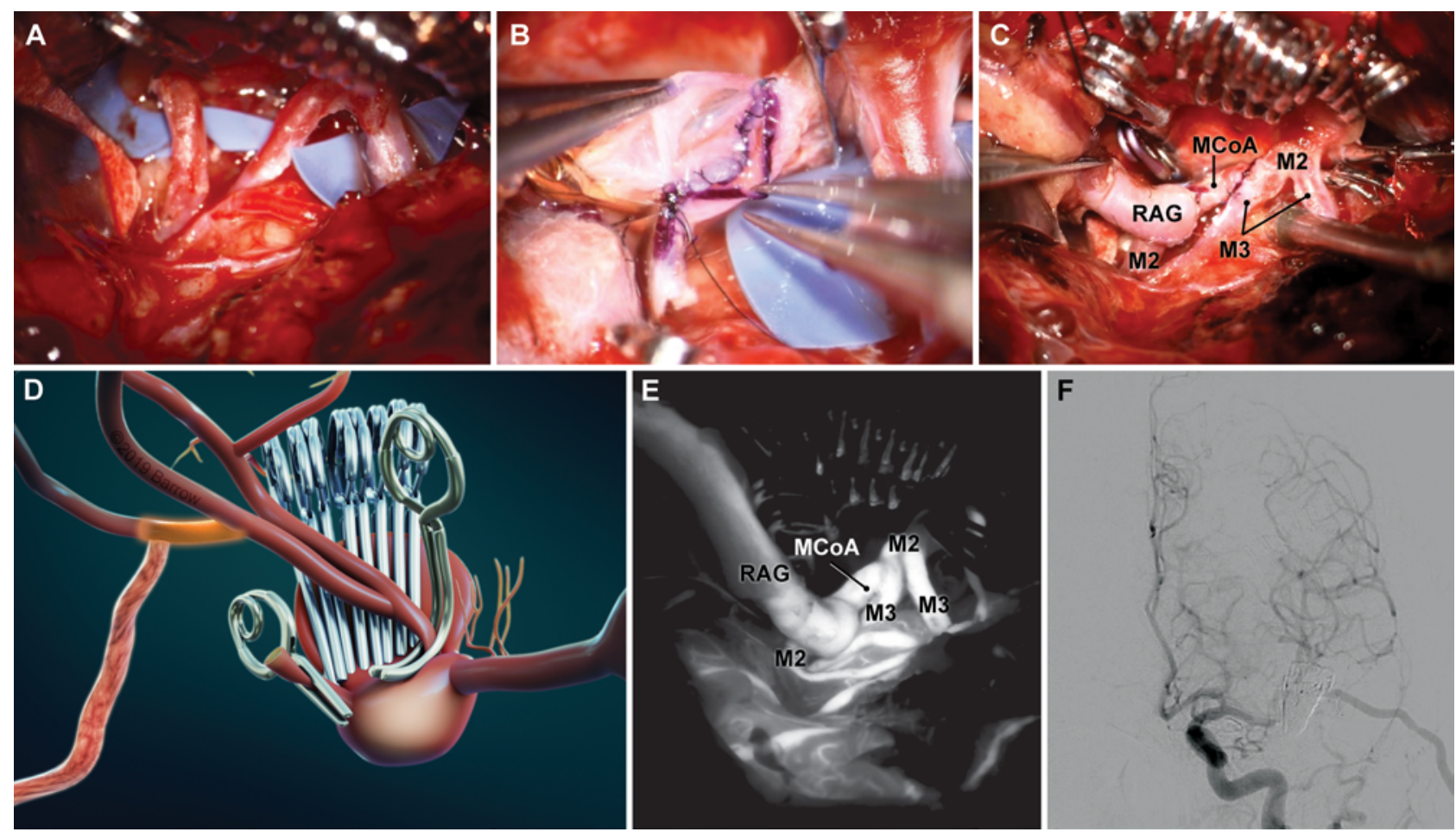

FIG. 4. Case 2. A: Intraoperative photograph demonstrating the temporal division of $\mathrm{M}_{2}$ and early bifurcation of the frontal division of $\mathrm{M}_{2}$. B: Intraoperative photograph of the back wall of the RAG-M $\mathrm{M}_{2}$ anastomosis performed using an intraluminal technique. C: Intraoperative photograph demonstrating the $\mathrm{MCoA}$ after end-to-side anastomosis of the temporal and frontal divisions. D: Illustration of the novel angioarchitecture after distal clip occlusion of the aneurysm, with the MCoA highlighted. E: Intraoperative ICG angiography demonstrating patency of two anastomoses. F: Postoperative common carotid artery injection DSA revealing obliteration of the aneurysm with preservation of the $\mathrm{M}_{1}$ lenticulostriate vessels and distal MCA flow through the high-flow bypass. Panels A-D are used with permission from Barrow Neurological Institute, Phoenix, Arizona.

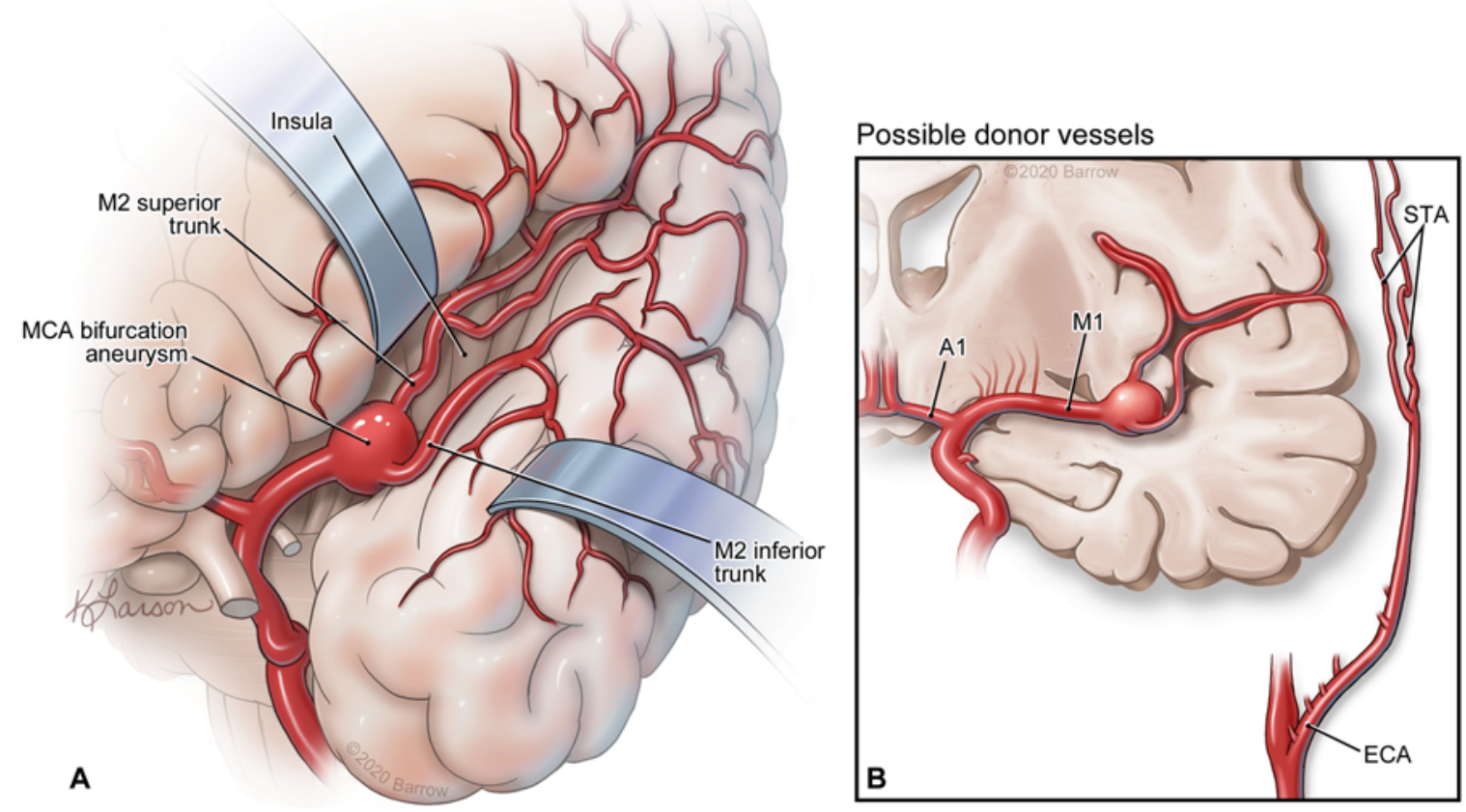

FIG. 5. Illustration showing donor arteries available for the bypass and their relationship to the MCA bifurcation aneurysm. A: The efferent $\mathrm{M}_{2}$ trunks lie close, allowing for a combination of bypass techniques. A wide sylvian fissure dissection is required to expose the aneurysm and its branches. B: In the cases presented, the STA and the ECA were selected as donors, but $A_{1}$ and $M_{1}$ are also viable intracranial options, and the common carotid artery is another extracranial option. Used with permission from Barrow Neurological Institute, Phoenix, Arizona. 

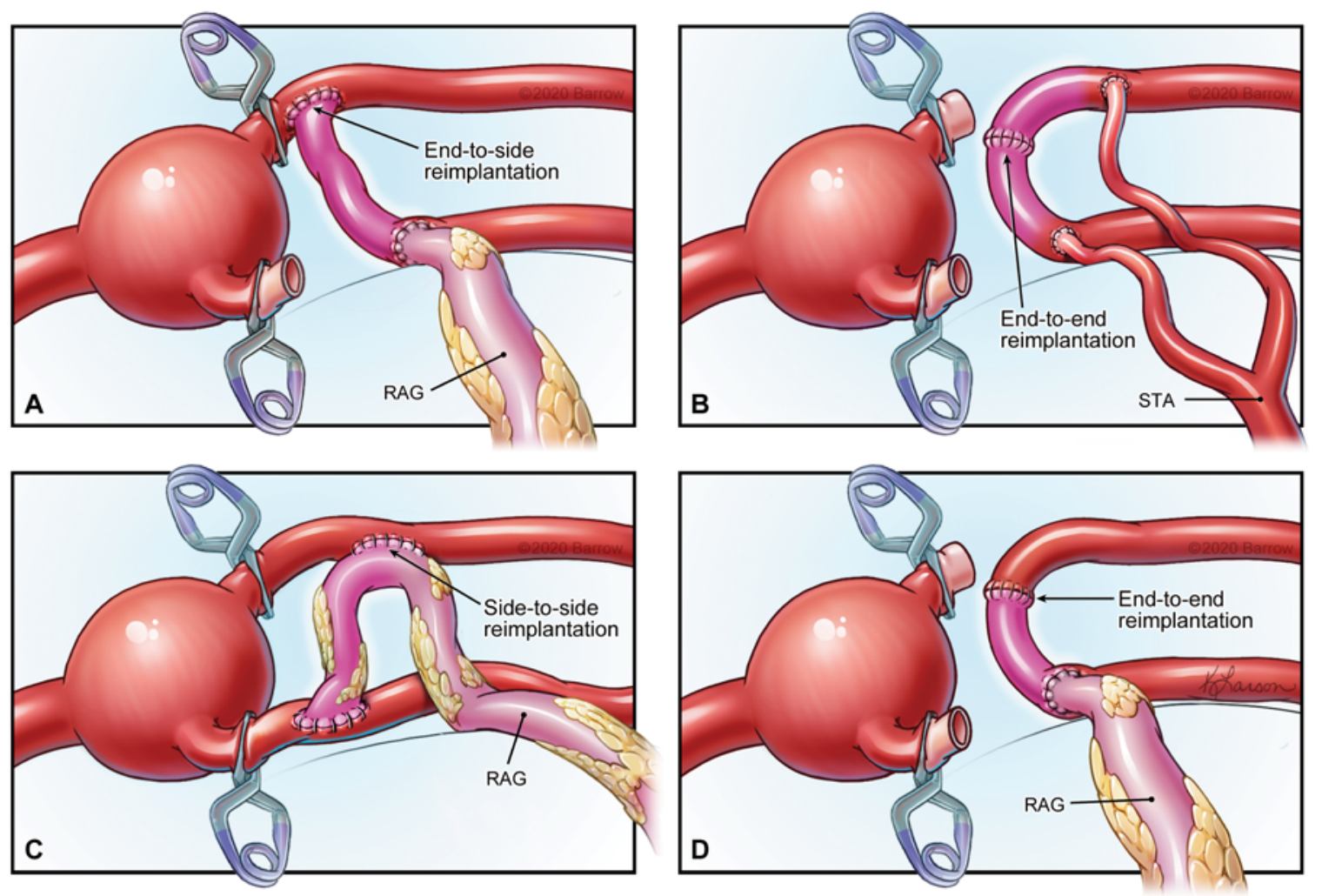

FIG. 6. Illustration showing the variety of combination bypasses available to create the MCoA (pink). A: After anastomosis of the donor to the inferior trunk, the proximal stump is reimplanted end-to-side to the superior trunk. B: Double-barrel STA-M ${ }_{2}$ bypass followed by an end-to-end reimplantation. C: Double reimplantation of the $\mathrm{M}_{2}$ branches onto the donor graft with side-to-side and end-to-side anastomoses. D: Similar to the illustration in A, but with an end-to-end reimplantation rather than end-to-side, and similar to the illustration in B, but with an EC-IC interpositional bypass rather than a double-barrel STA-M $\mathrm{M}_{2}$ bypass. Used with permission from Barrow Neurological Institute, Phoenix, Arizona.

cortical recipient arteries; ${ }^{8}$ second-generation EC-IC interpositional bypasses use the same end-to-side anastomoses, but an interposition graft tunneled from the cervical carotid artery to the intracranial recipient yields high bypass flow; ${ }^{9}$ and third-generation bypasses are intracranial reconstructions that reanastomose parent arteries, reimplant efferent branches, revascularize efferent branches with in situ donor arteries, and jump between donor and recipient with an interpositional graft. ${ }^{10}$ Reimplantation joins an efferent branch deliberately detached from an aneurysm to an adjacent artery with an end-to-side anastomosis, whereas in situ bypass joins donor and recipient arteries that run parallel and in proximity to one another with a side-to-side, "one-way-up" anastomosis that eliminates the need for arterial mobility. Reanastomosis rejoins transected ends of afferent and efferent arteries after excising the diseased section to restore parent artery anatomy and flow. The IC-IC interpositional bypass is an alternative connecting two arteries with a jump graft when tension on transected arterial ends is too great for reanastomosis or when the donor is out of reach for reimplantation or in situ bypass.

Fourth-generation bypasses use combinations of unconventional structure and unconventional suturing techniques (Fig. 6). For example, reattaching an efferent artery to a parent or adjacent artery is conventionally done with an end-to-side anastomosis (third-generation reimplantation) but can also be done unconventionally with an end-to-end anastomosis (fourth-generation reimplantation, as in case 1). Similarly, afferent and efferent arteries are conventionally joined with end-to-end anastomosis (third-generation reanastomosis) but can also be done unconventionally with an end-to-side anastomosis (fourth-generation reanastomosis). ${ }^{3}$ Additionally, end-to-side or end-to-end anastomosis is conventionally done with the use of an extraluminal suturing technique but can also be done unconventionally with an intraluminal suturing technique for the back wall without an actual side-to-side anastomosis (fourth-generation technique, as in case 2). The MCoAs in this report were both fourth-generation bypasses, as described above.

Other MCoAs were considered in these cases, including a second-generation ECA-RAG- $\mathrm{M}_{2}+\mathrm{M}_{2}$ bypass and a fourth-generation $A_{1}-R A G-M_{2}+M_{2}$ bypass. In case 2 , the former double reimplantation bypass was abandoned when the RAG was too short for the second reimplantation. The $\mathrm{MCoA}$ construct proved to be a versatile alternative to overcome the short RAG graft.

\section{Ischemia in Combination Bypasses}

The MCoA model is ideally suited for rebuilding biand trifurcated anatomy. Nonetheless, one of the dangers of double and triple bypasses is prolonged ischemia during 

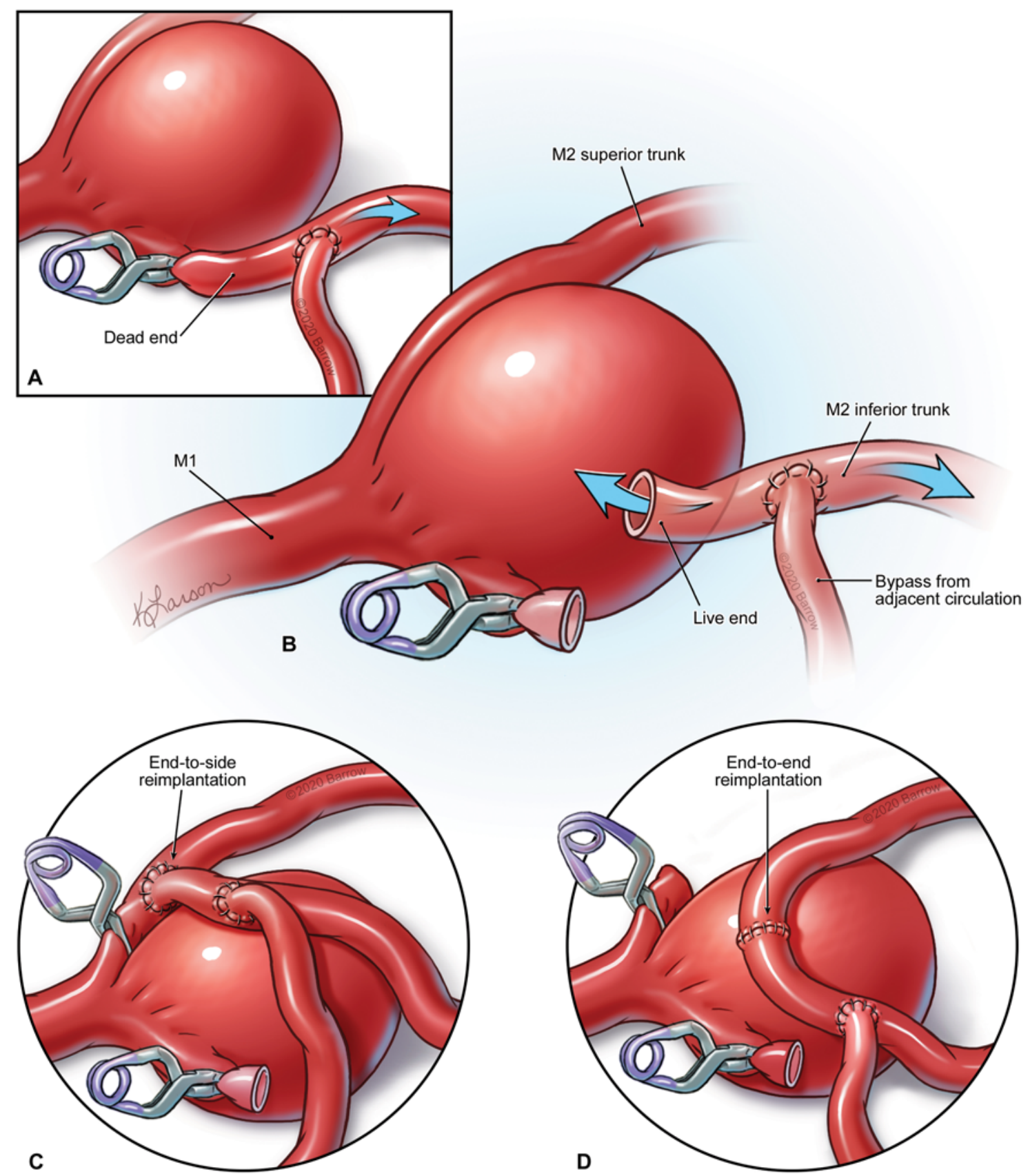

FIG. 7. Illustrations showing repurposing of the inferior trunk after distal occlusion of the aneurysm. Blue arrows show the direction of blood flow. After distally occluding the aneurysm's inferior trunk, the proximal stump is a dead end with no flow (A). This dead end can be transected and repurposed as a live end to arterialize the adjacent circulation (B). This circulation can be reestablished with either an end-to-side (C) or end-to-end (D) anastomosis, without additional ischemia time to the territory of the inferior trunk. Used with permission from Barrow Neurological Institute, Phoenix, Arizona.

multiple periods of temporary occlusion. For example, if the MCA trunks were joined before the EC-IC bypasses in either of our patients, then each trunk would have been subjected to two ischemic periods. However, creating the MCoA after tapping the ECA circulation resulted in only one ischemic period per trunk. Therefore, the ischemic dangers of combination bypass can be eliminated with careful sequencing and construction. Our MCoA constructs demonstrate the concept of donor reimplantation, which flips the usual recipient relationship of a reimplanted artery. When the MCA trunk is revascularized with either an STA-MCA or an ECA-RAG-M ${ }_{2}$ bypass, the recipient artery is supplied distally with the anterograde flow, and the proximal segment of the artery between the anastomosis and the distal trapping clip is a dead end. In our patients, however, it was repurposed as a live end to supply the MCoA (Fig. 7). In case 1, both proximal $\mathrm{M}_{2}$ trunks were live ends used as reimplanted donors after transec- 
tion from the aneurysm. In case 2, the proximal temporal $\left(\mathrm{M}_{2}\right)$ trunk was the live end. Donor reimplantation of the proximal $\mathrm{M}_{2}$ trunks enables the MCoA construction without ischemia because the distal $\mathrm{M}_{2}$ flow is uninterrupted. The end-to-end anastomosis in case 1 produced no ischemia time whatsoever, and the end-to-side anastomosis in case 2 produced ischemia time only in the frontal trunk. Therefore, construction of the MCoA reduces ischemia time, potentially improving safety, with ischemia time for the entire construct equal to just one period for each of the two $\mathrm{M}_{2}$ trunks. Compare this with an $\mathrm{M}_{2}-\mathrm{M}_{2}$ side-toside bypass, or an arborization bypass, which would have required an ischemic period in the EC-IC recipient trunk and another ischemic period in both $\mathrm{M}_{2}$ trunks (entire territory) for an in situ bypass.

\section{Conclusions}

This report introduces the concept of the MCoA construct and demonstrates two of its many variations. Angioarchitectural and technical elements include the donation of flow from an adjacent circulation, a communicating bypass, application of fourth-generation bypass techniques, and a minimized ischemia time. The MCoA constructs are ideally suited for rebuilding bi- and trifurcated vascular anatomy after trapping or distally occluding complex MCA aneurysms. The MCoA also demonstrates that bypass surgery is a fertile field for innovative advances in vascular neurosurgery.

\section{Acknowledgments}

We thank the staff of Neuroscience Publications at Barrow Neurological Institute for assistance with manuscript preparation.

\section{References}

1. Tayebi Meybodi A, Benet A, Griswold D, et al. Anatomical assessment of the temporopolar artery for revascularization of deep recipients. Oper Neurosurg (Hagerstown). 2019;16(3):335-344.

2. Tayebi Meybodi A, Lawton MT, Griswold D, et al. Assessment of the temporopolar artery as a donor artery for intracranial-intracranial bypass to the middle cerebral artery: anatomic feasibility study. World Neurosurg. 2017;104:171-179.
3. Lawton MT, Lang MJ. The future of open vascular neurosurgery: perspectives on cavernous malformations, AVMs, and bypasses for complex aneurysms. J Neurosurg. 2019;130(5):1409-1425.

4. Lawton MT. Seven Bypasses: Tenets and Techniques for Revascularization. Thieme; 2018.

5. Davies JM, Lawton MT. "Picket fence" clipping technique for large and complex aneurysms. Neurosurg Focus. 2015;39 Video Suppl 1:V17.

6. Tayebi Meybodi A, Huang W, Benet A, et al. Bypass surgery for complex middle cerebral artery aneurysms: an algorithmic approach to revascularization. J Neurosurg. 2017;127(3):463-479.

7. Wessels L, Fekonja LS, Vajkoczy P. Bypass surgery of complex middle cerebral artery aneurysms-technical aspects and outcomes. Acta Neurochir (Wien). 2019;161(10):1981-1991.

8. Yaşargil MG. Microneurosurgery. Vol 1. Thieme; 1984.

9. Sundt TM Jr, Piepgras DG, Houser OW, Campbell JK. Interposition saphenous vein grafts for advanced occlusive disease and large aneurysms in the posterior circulation. J Neurosurg. 1982;56(2):205-215.

10. Sanai N, Zador Z, Lawton MT. Bypass surgery for complex brain aneurysms: an assessment of intracranial-intracranial bypass. Neurosurgery. 2009;65(4):670-683.

\section{Disclosures}

The authors report no conflict of interest concerning the materials or methods used in this study or the findings specified in this paper.

\section{Author Contributions}

Conception and design: Lawton, Frisoli. Acquisition of data: Frisoli, Catapano, Baranoski. Analysis and interpretation of data: all authors. Drafting the article: Frisoli, Catapano, Baranoski. Critically revising the article: Lawton. Reviewed submitted version of manuscript: all authors. Study supervision: Lawton.

\section{Correspondence}

Michael T. Lawton: c/o Neuroscience Publications, Barrow Neurological Institute, St. Joseph's Hospital and Medical Center, Phoenix, AZ.neuropub@barrowneuro.org. 